\title{
Long term dynamical evolution of the Oort cloud comets: galactic and planetary perturbations
}

\author{
Piotr A. Dybczyński \\ Astronomical Observatory of the A. Mickiewicz Univ., Poznań, Poland \\ e-mail: dybol@amu.edu.pl
}

\begin{abstract}
The source of long-period comets can be numerically modeled by means of Monte Carlo simulation of the Oort cloud dynamics. Tracing a comet motion under the galactic perturbations over a long time interval requires taking into account of planetary perturbations. A method for the approximate treating of the planetary perturbations in such simulations is described. Some peculiarities found in planetary action on long-period simulated cometary sample are also discussed.
\end{abstract}

Keywords. Comets: general, Oort Cloud.

\section{Introduction}

The source of the observed long period comets may be modeled numerically with the Monte Carlo simulation of the long term dynamical evolution of the bodies in the Oort cloud under the influence of stellar and Galactic perturbations. In this paper we concentrate on the influence of the planetary perturbations during the cometary perihelion passages among the Solar System planets. It is very expensive (in CPU time) to account for planetary perturbations in an exact manner (e.g. by numerical integration of motion) because the simulation typically extends for $10^{6}-10^{9}$ years. In a highly simplified case one can assume either that each comet is removed from the observable part of the cloud after its first perihelion passage below the observability limit $(O L)$, or that planetary perturbation might be completely ignored, thus allowing a comet to make several, even hundreds of observable perihelion passages without any change to its orbit.

As it was shown recently by Dybczyński (2004) both the above-mentioned approaches are far from the truth. A convenient solution has been proposed: the use of the Planetary System transparency coefficient $P$, defined as the probability that the planetary perturbations remove a particular comet from the observable part of the cloud during a single perihelion passage among planets.

Before we go into details let us recall the typical orbit evolution of an Oort cloud comet under the Galactic disk tide. Such an evolution for the case of $a=20000 \mathrm{AU}$ is presented in Fig. 1. In this kind of evolution the argument of perihelion $\omega$, the inclination $i$ and perihelion distance $q$ evolve in a strictly synchronous manner: the minima of $q$ and $i$ coincide and $\omega$ crosses the $270^{\circ}$ or $90^{\circ}$ value at the same moment. The vertical lines in Fig. 1 represent changes in the heliocentric distance of a comet. Each vertical line marks one perihelion passage (due to the scale of the horizontal axis the descending and ascending branches overlap).

Planetary perturbations limit the number of consecutive perihelion passages of a particular comet and as a result they significantly decrease the new comet influx. Additionally, 


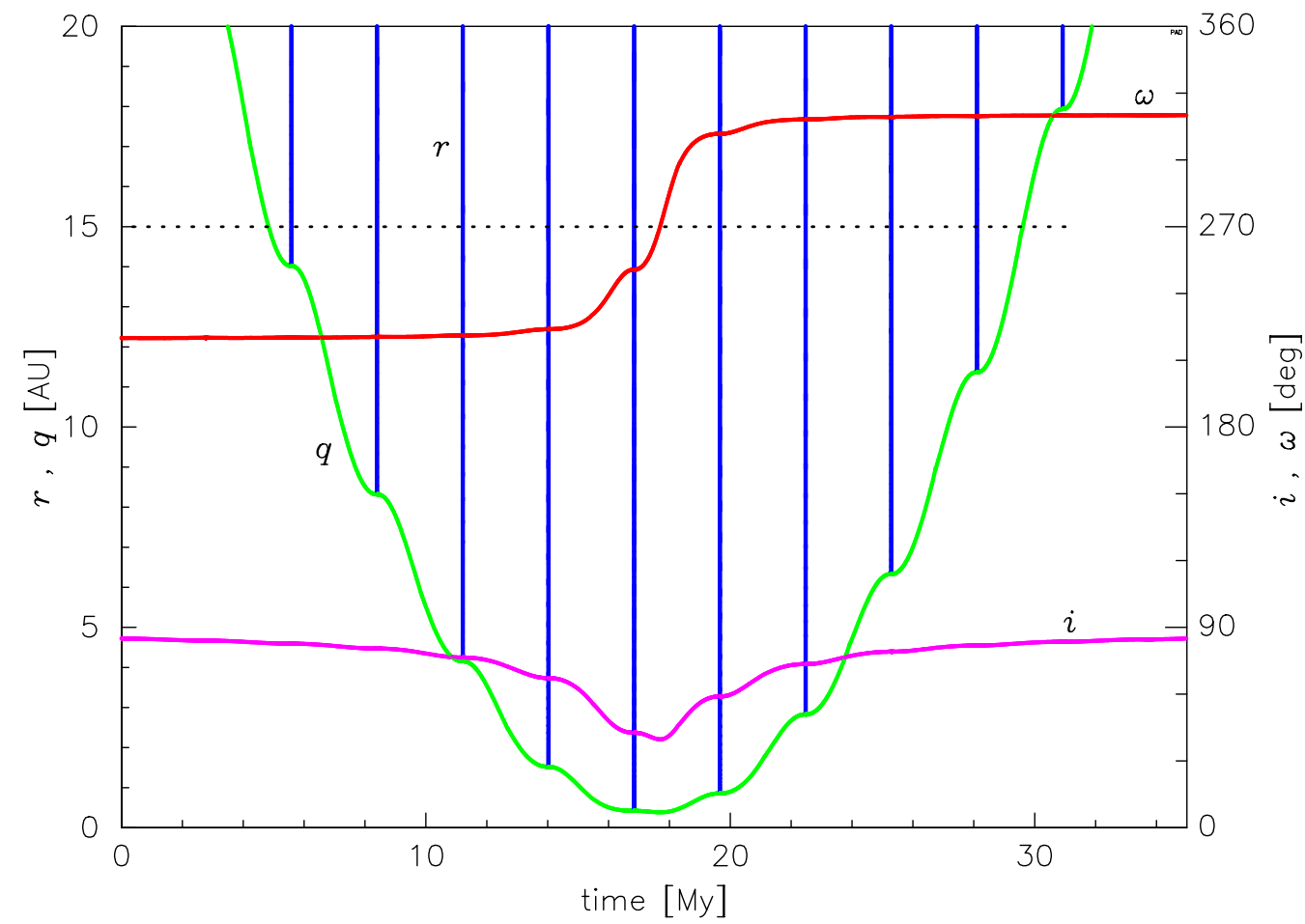

Figure 1. An example of an orbit evolution under the influence of the Galactic disk tide. In this plot the simultaneous long term evolution of the inclination $i$ and the argument of perihelion $\omega$ (both in degrees, see right hand vertical axis) as well as perihelion distance $q$ in AU (left hand vertical axis) is presented. Additionally, the changes in the cometary heliocentric distance $r$ are plotted, which produces a series of vertical lines due to the relatively short time spans spent in the inner solar system (below $20 \mathrm{AU}$ ). Note that during a single minimum of the perihelion distance this comet crosses the adopted planetary perturbation limit at 15 AU (dotted line) 9 times. Here the semi-major axis $a=20000$ AU.

due to the strictly synchronous changes in the orbital elements, their distributions depend on the strength of the planetary effect on a comet.

\section{Planetary System transparency coefficient}

Dybczyński (2004) estimated the value of the coefficient $P$ on the basis of the observed, cloned and simulated samples of the Oort cloud comets. The method of estimation is based on the comparison of cometary orbits (especially the perihelion distances) one orbital revolution into the future, calculated twice, one with planetary perturbations completely omitted and the other one with perturbations fully taken into account. The dynamical model of the cometary motion among Solar System planets is based on the JPL DE406 planetary ephemerides (Standish 1998) and fully described in Dybczyński (2001). A special technique of comet cloning is proposed to obtain more valuable $P$ estimations based on much more numerous cometary sample. An additional check is made by estimating the planetary system transparency coefficient from synthetic, simulated long-period observable comets. The are obtained are almost all in good agreement as shown in Table 1 . The discrepancy between $P=0.64$ obtained from the observed sample 


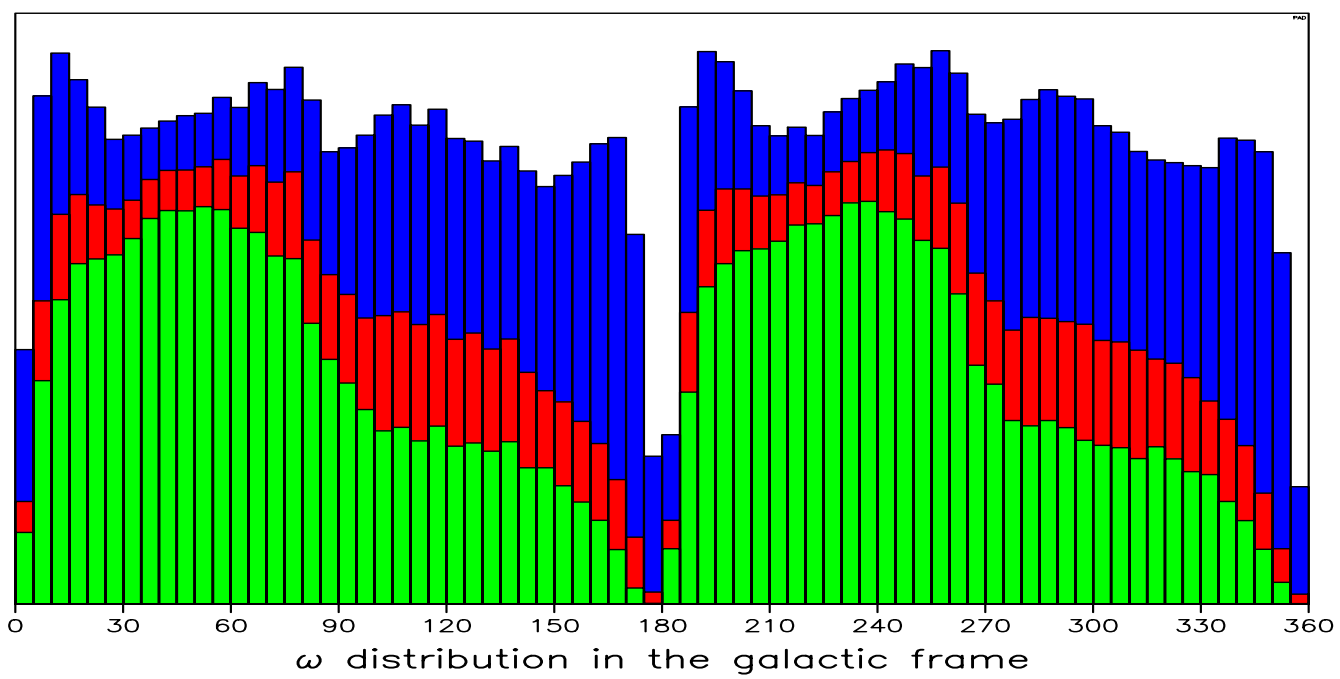

Figure 2. Three different (overlapped) distributions of the argument of perihelion of simulated observable comets with $a>20000 \mathrm{AU}$ for three different $P$ values: $P=0.05$ (dark-grey, background histogram), $P=0.50$ (grey histogram) and $P=0.95$ (light-grey, foreground histogram).

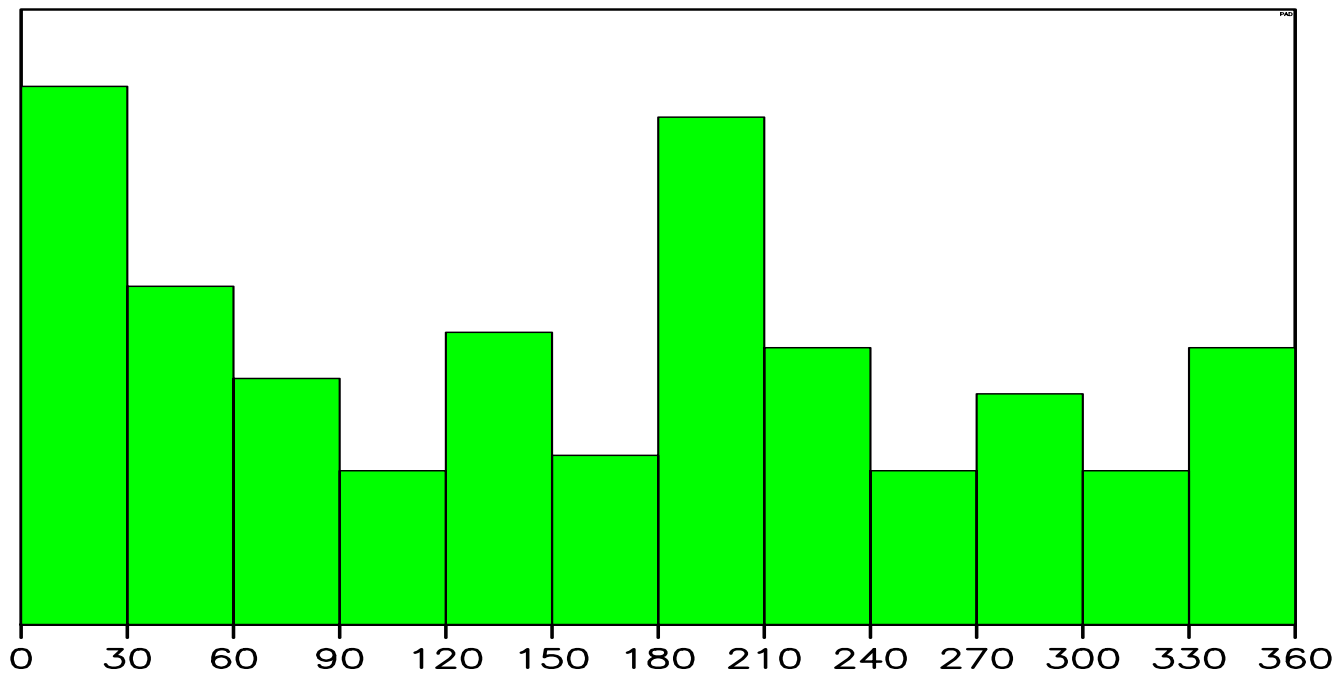

Figure 3. The distribution of the argument of perihelion with respect to the Galactic disk for 371 observed elliptic comets of classes 1 and 2, taken from the 15th edition of the Catalogue of Cometary Orbits(Marsden \& Williams 2003) .

and $P=0.48$ from the cloned and simulated ones is some bias resulting from the specific orientation of the elliptic cometary sample with respect to the Planetary System, as it is demonstrated and explained in detail in the above-mentioned paper.

The proposed approximation is a very convenient way of incorporating planetary perturbations into a dynamical model used in Oort cloud evolution simulations. In many cases it can be applied after the main simulation calculations, during the analysis stage of the project. Below we present two examples of the successful use of $P$ in the Oort cloud modeling. The simulation code is highly simplified in both cases (Galactic center and stellar perturbations omitted) thus the results presented in the next two sections are not intended to be compared with observations, they only illustrate the improvements 


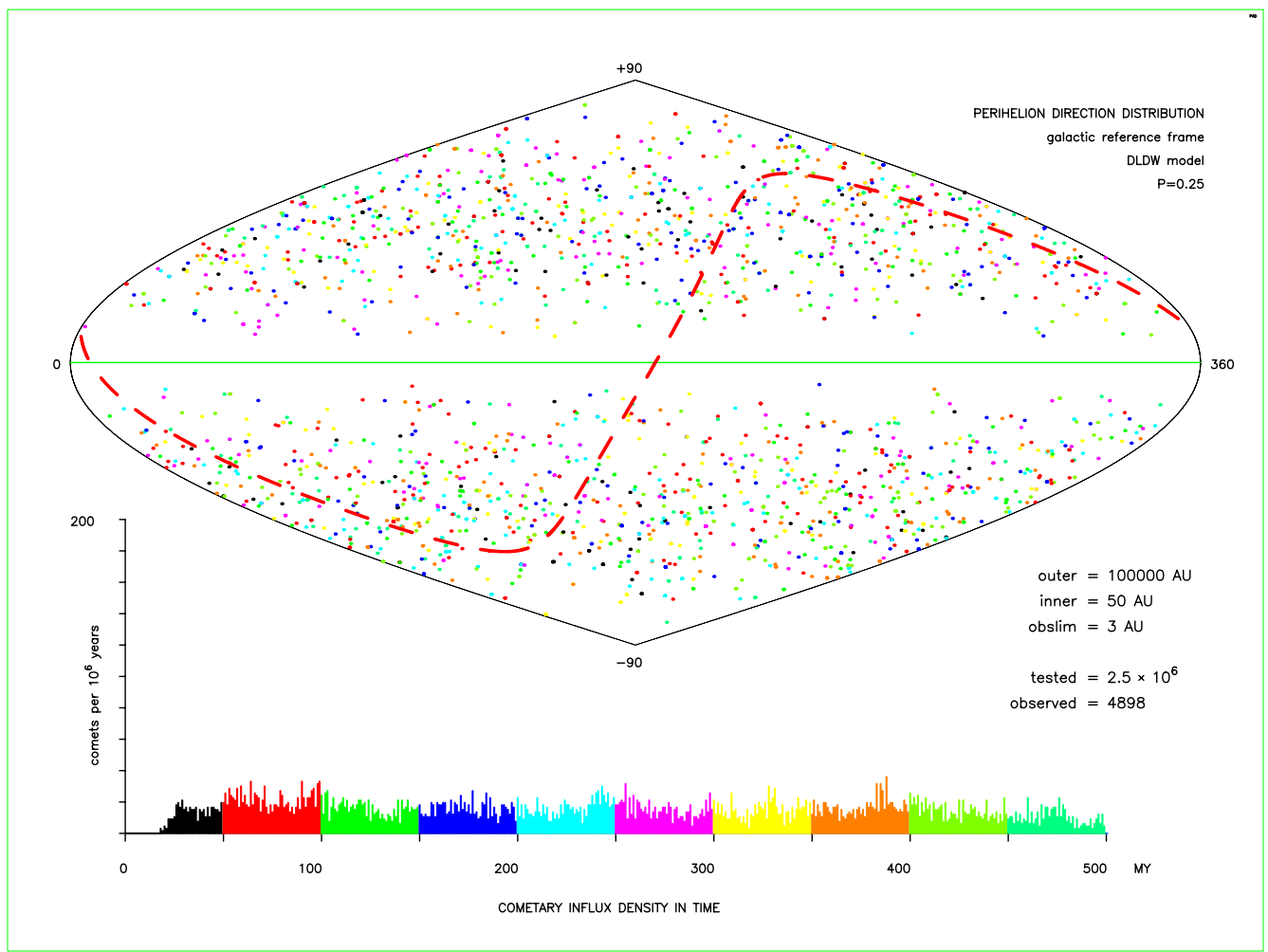

Figure 4. The perihelion direction distribution in galactic reference frame for comets with semi-major axis $a<20000 \mathrm{AU}$. The DLDW cloud model is flattened in its inner part towards the Solar System invariant plane (dashed curve in the main plot) which, combined with the orientation of the Galactic disk produces strong anisotropies. The overpopulation of these regions comes from ignoring planetary perturbations $(P=0.0)$.

Table 1. The Planetary System transparency coefficient $P$ for the observability limit $O L=3 \mathrm{AU}$, estimated from different cometary samples by Dybczyński (2004).

\begin{tabular}{ccc}
\hline Source of estimation & $a<10000 \mathrm{AU}$ & $a>10000 \mathrm{AU}$ \\
\hline observed comets & 0.23 & 0.64 \\
cloned comets & 0.24 & 0.48 \\
simulated comets & - & 0.49 \\
\hline
\end{tabular}

possible to achieve when using the Planetary System transparency coefficient $P$. In all these calculations we used the DLDW model of the Oort cloud, based on the unpublished results obtained by Dones, Levison, Duncan and Weissman; for details see Dybczyński (2002).

\section{Argument of perihelion distribution for simulated observable comets}

Due to a synchronous variations of cometary orbital elements under the Galactic disk tidal perturbations the simulated distribution of the argument of perihelion with respect to the Galactic frame strongly depends on the Planetary System transparency coefficient $P$. In Fig. 2 we present this dependence for the simulated observable comets with $a>$ 


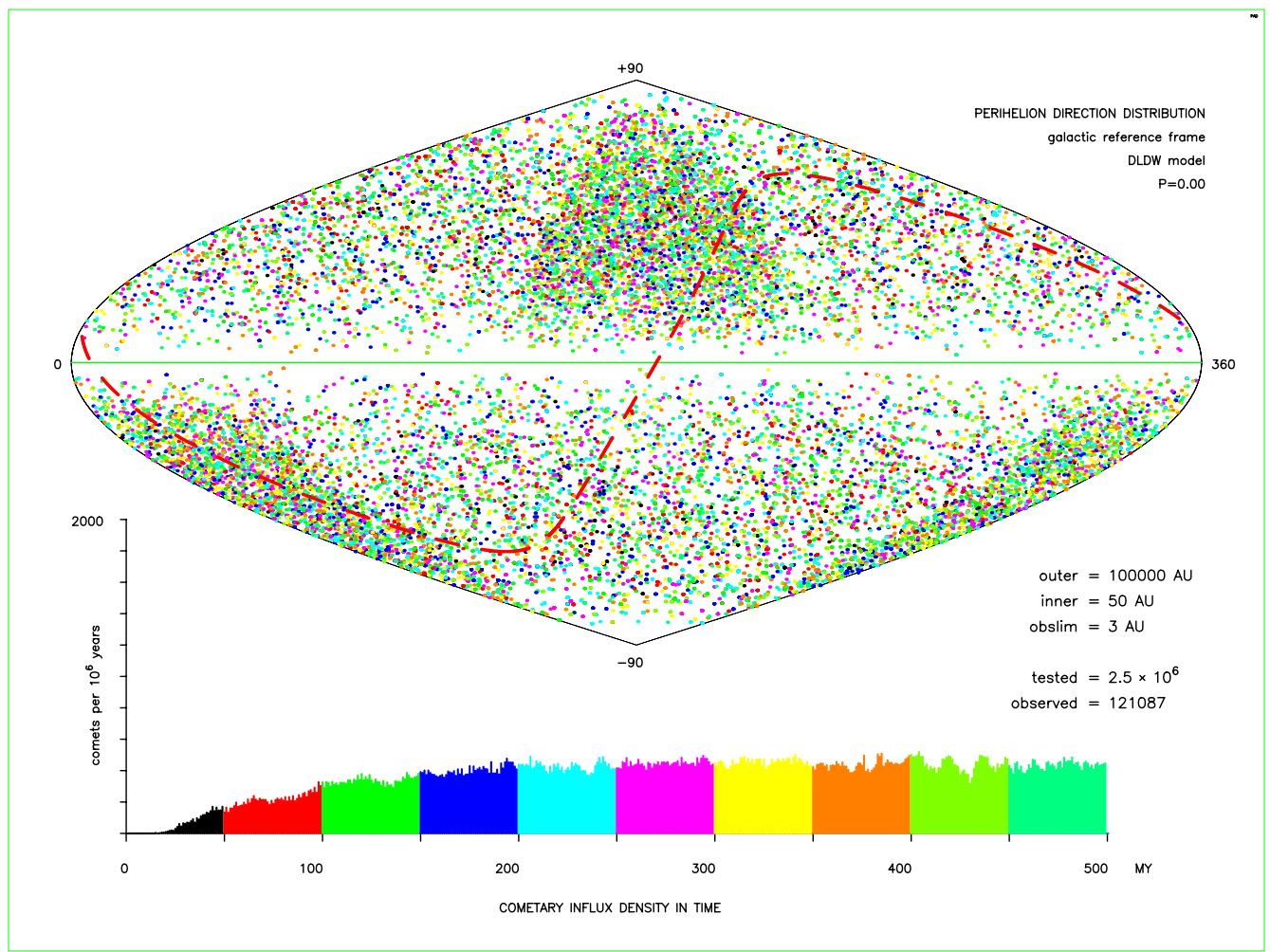

Figure 5. The same observable cometary sample as in Fig. 4 but after applying $P=0.25$. Artificial anisotropies and concentrations disappeared.

20 000AU. As it may be inferred from Fig. 1, the lower $P$ value gives strong preferences for the low semi-major axis comets, resulting in much more uniform $\omega$ distribution (except regions close to $0^{\circ}$ and $180^{\circ}$, which always remain empty in this simplified model). For higher $P$ values those comets are generally removed from the observed flux and the distribution is dominated by comets observed during the first (and sometimes the second) perihelion passage. As a result the first and the third quarters of the $\omega$ distribution are relatively more populated. Such an asymmetry is seen in the really observed long-period comets population as it is shown in Fig. 3. Comparing this to the distributions presented in Fig. 2 confirms that using $P \simeq 0.5$ produces much more reliable simulation output than $P \simeq 0$, what correspond simply to ignoring the planetary perturbations.

\section{Anisotropies for low semi-major-axes comets}

Another example of a successful use of the Planetary System transparency coefficient is the analysis of the directional distribution of the simulated observable comets with low semi-major-axes. We restrict here our consideration to comets with the semi-major axes $a<20000 \mathrm{AU}$, what typically constitute more than $50 \%$ of the long-period cometary influx. If we plot perihelion directions of these comets on a celestial sphere, we obtain the distribution shown in Fig. 4. The mutual orientation of the flattened inner part of the cloud in the DLDW model and the Galactic disk generates here two privileged regions, highly overpopulated when omitting planetary perturbations $(P=0)$. If we apply $P=$ 0.25 , recommended for small semi-major axis comets, we obtain the distribution shown in Fig. 5. Note that due to the dramatic decrease in the cometary flux, the vertical scale 
of the flux density in time has to be ten times enlarged. It should be stressed here that the concentrations shown in Fig. 4 are completely absent in the observed samples of orbits thus applying the nonzero value of $P$ brings the simulation output much closer to the reality. Additionally the overall number of the observed simulated comets decreased here from 121087 down to 4898 bodies. When scaled to the cloud population of $2 \times 10^{12}$ comets it means that the influx of the new comets decreased from over 400/year down to $\sim 20$ /year what is much closer to the estimations of the overall long-period comets influx for $O L=3 \mathrm{AU}$, see for example Wiegert \& Tremaine (1999).

\section{Conclusions}

The Planetary System transparency coefficient is shown to be a useful tool for including planetary perturbations into a dynamical model used in numerical simulations of a long time evolution of the Oort cloud comets. We demonstrated that the asymmetric distribution of the galactic argument of perihelion of the observed long-period comets with high quality orbits can be successfully reproduced when $P \simeq 0.5$ is used. We also showed that the nonzero $P$ value is necessary to remove some artificial directional concentrations of the simulated observable comets sample, especially for low semi-major axes comets from the flattened, inner part of the simulated Oort cloud. It has been also shown that the Solar System transparency coefficient $P$ influences both the geometrical characteristics of the simulated comets as well as the long-period cometary influx obtained from simulations. The proposed method of including the planetary perturbations into the dynamical model of the Oort cloud dynamics used in numerical simulations proved to be powerful while still very simple. We incorporate this approximation into the extended simulation computer code for the long-term Oort cloud comets dynamical evolution under the influence of stellar and Galactic perturbations. This work is in progress.

\section{Acknowledgements}

The author is indebted to an anonymous referee for comments and suggestions which allowed to improve the quality of this manuscript. The research described in this paper was supported by KBN grant no. 2P03D01324.

\section{References}

Dybczyński, P. A. 2001, Astron. Astrophys. 375, 643

Dybczyński, P. A. 2002, Astron. Astrophys. 396, 283

Dybczyński, P. A. 2004, Astron. Astrophys. in press

Marsden, B. G. \& Williams, G. V. 2003, Catalogue of Cometary Orbits, 15th Edition (Cambridge, Mass.: Smithsonian Astrophysical Observatory)

Standish, E. M. 1998, JPL Planetary and Lunar Ephemerides, Interoffice Memorandum IOM 312.F - 98 - 048, Jet Propulsion Laboratory

Wiegert, P. \& Tremaine, S. 1999, Icarus 137, 84 